\title{
Recursive multi-SEs NWTH method for small target detection in infrared images
}

\author{
Tae-Wuk Bae ${ }^{1 \mathrm{a})}$, Byoung-Ik Kim ${ }^{1}$, Fei Zhang ${ }^{2}$, \\ Young-Choon Kim ${ }^{3}$, Sang-Ho Ahn ${ }^{4}$, and Kyu-Ik Sohng ${ }^{1}$ \\ ${ }^{1}$ School of Electrical Engineering and Computer Science, Kyungpook National Uni- \\ versity, Buk-Gu, Daegu, 702-701, Korea \\ ${ }^{2}$ Department of Electrical and Computer Engineering, Michigan State University, \\ USA \\ ${ }^{3}$ Dept. of Information and Communication Engineering, Youngdong University, \\ Korea \\ ${ }^{4}$ School of Electronic Telecommunication, Inje University, Korea \\ a)nanninggo@gmail.com
}

\begin{abstract}
Mathematical morphology techniques, such as white tophat (WTH) and new white top-hat (NWTH) transformation have been researched for small target detection application in the infrared (IR) images. However, its target enhancement performance still depends on its SE size set by users. To solve this problem, we propose a recursive multi-structuring elements (multi-SEs) NWTH method with an automatic decision mechanism of the SE size. The proposed method based on the NWTH transformation updates the multi-SEs by calculating candidate target-to-clutter ratio gain (CTCRG) of the NWTH images by the multi-SEs. Through a recursive procedure, final multi-SEs is automatically selected, and then small targets can be detected in a summation image of NWTH images by the final multi-SEs.
\end{abstract}

Keywords: small target, top-hat, structuring element, IR

Classification: Microwave and millimeter wave devices, circuits, and systems

\section{References}

[1] S. D. Deshpande, M. H. Er, V. Ronda, and P. Chan, "Max-mean and max-median filters for detection of small-targets," Proc. SPIE, vol. 3809, pp. 74-83, 1999.

[2] T. Zhang, Z. Zuo, W. Yang, and X. Sun, "Moving dim point target detection with three-dimensional wide-to-exact search directional filtering," Pattern Recognition Letters, vol. 28, no. 2, pp. 246-253, 2007.

[3] P. A. Ffrench, J. R. Zeidler, and W. H. Ku, "Enhanced detectability of small objects in correlated clutter using an improved 2-D adaptive lattice algorithm," IEEE Trans. Image Process., vol. 6, no. 3, pp. 383-397, March 1997.

[4] P. Jackway, "Improved morphological Top-hat," IEEE Electron. Lett., vol. 36, no. 14, pp. 1194-1195, July 2000. 
[5] X. Bai, F. Zhou, and Y. Xie, "New class of top-hat transformation to enhance infrared small targets," J. Electronic Imaging, vol. 17, no. 3, pp. 0305011-3, 2008.

[6] X. Mao and W. Diao, "Criterion to evaluate the quality of infrared small target images," Int. J. Infrared Millim. Waves, vol. 30, no. 1, pp. 56-64, 2008.

[7] C. I. Hilliard, "Selection of a clutter rejection algorithm for real-time target detection from an airborne platform," Proc. SPIE, vol. 4048, pp. 74-84, 2000 .

\section{Introduction}

Because of dim intensity and ambiguous shape of small targets, it is a challenging task to detect the infrared (IR) small target under background with heavy clutter. Various methods $[1,2,3,4,5]$ were proposed to enhance the small target embedded in heavy clutter. However, max-mean filter [1] and max-median filter [1] is sensitive to clutter distribution or variation of target features. 3D directional filter [2] needs several consecutive images that contain target motion, but the images are not easy to be obtained. 2D adaptive lattice algorithm [3] can be used to suppress clutter, but is complex. Most of those methods mentioned above are complex or time consuming or ineffective under the conditions of heavy clutter and dim target.

For the parallel processing and easy implementation in real-time system, top-hat (TH) transformation [4] is widely used to enhance small targets. However, due to various clutter distribution and smoothing property of image detail by morphological operations, the target enhancement performance is weak. Besides, user must set its structuring element (SE) size with prior information about the target size. Recently, new white top-hat (NWTH) transformation [5] modifying white top-hat (WTH) transformation was proposed. Because of its SE structure, it has better target enhancement performance compared to the WTH transformation. However, its performance, also, is still affected by the decision of the SE size. To solve this problem, an automatic decision mechanism of the SE size is needed in the TH based algorithm. The proposed method updates the size of multi-structuring elements (multi-SEs) by calculating candidate target-to-clutter ratio gain (CTCRG) of the NWTH images by the multi-SEs. Through a recursive procedure, final multi-SEs is automatically selected, and then small targets can be detected in a summation image of NWTH images by the final multi-SEs.

\section{WTH and NWTH transformation}

Let $f$ and $B$ represent a gray image and a structuring element (SE), respectively. The opening, and WTH [5] of $f(x, y)$ by $B(i, j)$, denoted by, $f \circ B$, and WTH are defined by

$$
\begin{aligned}
& f \circ B=(f \Theta B) \oplus B \\
& \operatorname{WTH}(x, y)=f(x, y)-f \circ B(x, y)
\end{aligned}
$$




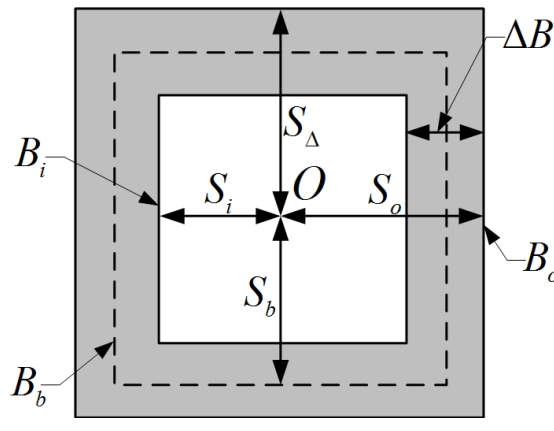

(a)

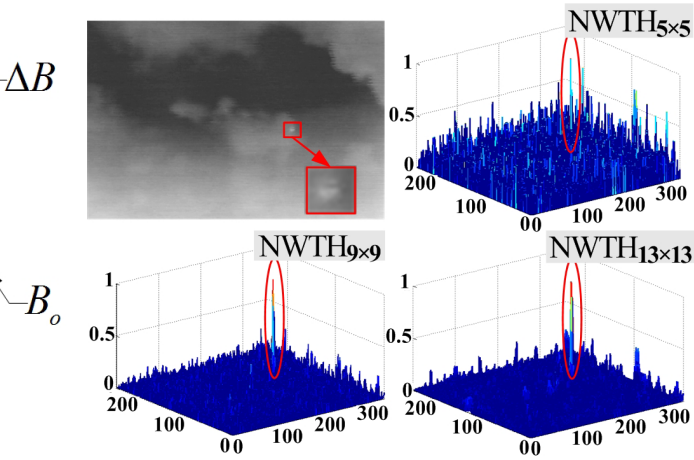

(b)

Fig. 1. (a) Relationship of structuring elements (SEs) in NWTH and (b) impact of SE size.

Where $f \Theta B$ and $f \oplus B$ represents the erosion and dilation of $f(x, y)$ by $B(i, j)$. Because erosion (dilation) makes the image gray value smaller (larger) than that of $f$, opening smoothes bright small regions of $f$. So, the WTH transformation can be used to detect the potential small target region in $f$.

Recently, to greatly improve the performance of the WTH transformation in small target application, the new white top-hat (NWTH) [5] was designed as follows:

$$
\begin{aligned}
& \operatorname{NWTH}(x, y)=f(x, y)-f \square B_{o i}(x, y) \\
& f \square B_{o i}(x, y)=(f \oplus \Delta B) \Theta B_{b}
\end{aligned}
$$

where $B_{o i}$ represents $B_{i}$ and $B_{o}$, inner and outer SE, two flat SEs with the same shape. The relationship of $B_{i}, B_{o}, B_{b}$ and $\Delta B$ is showed in Fig. 1 (a), in which $O$ is the origin of all the SEs. $S_{i}, S_{o}, S_{b}$ and $S_{\Delta}$ represents the size of $B_{i}, B_{o}, B_{b}$, and $\Delta B . B_{b}$ represents the $\mathrm{SE}$ whose size is between $S_{i}$ and $S_{o} . \Delta B$ is the margin region between $S_{i}$ and $S_{o}$. To make NWTH perform well in IR small target enhancement, some parameters was defined as follows: $S_{o}-S_{i}=1, S_{b}=S_{o}$. Fig. 1 (b) shows result images of NWTH transformation $\left(\mathrm{NWTH}_{5 \times 5}, \mathrm{NWTH}_{9 \times 9}\right.$, and $\left.\mathrm{NWTH}_{13 \times 13}\right)$ by various SE size, $5 \times 5\left(S_{o}=3\right)$, $9 \times 9\left(S_{o}=5\right)$, and $13 \times 13\left(S_{o}=7\right)$. If the SE size is much smaller than the target size as in the case of a $\mathrm{NWTH}_{5 \times 5}$ image, Eq. (4) can not replace the pixels of the target region with the pixels of surrounding regions. So, the NWTH transformation of Eq. (3) failed to enhance the small target region. On the other hand, if the SE size is much larger than the target size as in the case of a $\mathrm{NWTH}_{13 \times 13}$ image, Eq. (4) replace the pixels of surrounding regions near to cloud edge with the pixels of the cloud edge regions. So, the NWTH transformation of Eq. (3) brought about serious clutters on the cloud edge regions. And we can show that the NWTH transformation using the SE size of $9 \times 9$ (similar to the target size) has better target enhancement and background suppression performance. As mentioned, the decision of the SE size is an important factor in the target enhancement by the TH based algorithm. But we can not set the SE size under absence of prior information about a target size. So, the TH based algorithm needs an automatic decision 
mechanism of the SE size.

\section{Proposed recursive multi-SEs NWTH method}

In order to solve the mentioned detect of the NWTH transformation, we propose the recursive multi-SEs NWTH method with an automatic decision mechanism of the SE size. The proposed method has largely 3 steps, multiSEs NWTH step, candidate target-to-clutter ratio gain (CTCRG) step, and multi-SEs adjustment step. In the IR images, the detection problem of the small target is such that to detect the targets whose sizes are less than $0.15 \%$ of the whole image [6]. In case of an image of $128 \times 128$, the sizes of its small targets are ranging from $1 \times 1$ to $6 \times 6$. So, we assumed that the size of small targets is less than $15 \times 15$ considering the IR image size and visible target size. The size of initial multi-SEs is based on this reason.

At first, the multi-SEs NWTH images $\left(\mathrm{NWTH}_{a \times a}, \mathrm{NWTH}_{b \times b}\right.$, $\mathrm{NWTH}_{c \times c}$ ) is calculated for $a \times a, b \times b, c \times c$, three SEs (multi-SEs) with si $(=b-a=c-b)$, the SE interval. The SE interval means the size difference of sides between two adjacent SEs. In the recursive process, a base SE means the SE of a NWTH image having the highest target enhancement performance among the multi-SEs NWTH images. And a parameter $s z$ represents the size of one side of the base SE. If the base SE is accurately converged and si is more decreased by recursive process, target region in the multi-SEs NWTH images can be more prominent comparing to background region. To select the base SE among the multi-SEs, the CTCRG, the brief modification of the signal-to-clutter ratio gain (SCRG) [7] is calculated for the multi-SEs NWTH images as follows:

$$
C T C R G=\bar{T} / \sigma_{c}^{2}
$$

where $\bar{T}$ is the mean of the candidate target pixels and $\sigma_{c}^{2}$ is the variance of the background clutter pixels. The CTCRG is an automatic mechanism updating the multi-SEs for an IR image. To obtain final multi-SEs, the entire procedure for the proposed method is performed as follows.

Step 1: Set initial multi-SEs and initial si;

Step 2: Calculate multi-SEs NWTH images $\left(\mathrm{NWTH}_{a \times a}, \mathrm{NWTH}_{b \times b}\right.$, $\mathrm{NWTH}_{c \times c}$ ) through the multi-SEs with the si for an original IR image;

Step 3: Obtain $M_{s i}$, the summation image of the multi-SEs NWTH images at $s i$

Step 4: Find $M_{s i \_m a x}$, the maximum value in $M_{s i}$, and pixel coordinates more than $m \times M_{\text {si_max }}(m<1)$ in $M_{s i}$ are regarded as candidate target pixels and the residue pixel coordinates are regarded as background clutter pixels respectively in $M_{s i}$;

Step 5: Go to Step 8 if $s i=1$, otherwise go to Step 6; 
Step 6: Calculate respective CTCRG values $\left(\mathrm{CTCRG}_{a \times a}, \mathrm{CTCRG}_{b \times b}\right.$, $\mathrm{CTCRG}_{c \times c}$ ) by applying the pixel coordinates information of step 4 to the multi-SEs NWTH images;

Step 7: Select the SE of a NWTH image having the highest CTCRG value among the multi-SEs NWTH images as a base SE. By using $s z$ of the base SE, Set $s i=s i / 2$ and $a=s z-s i / 2, b=s z, c=s z+s i / 2$ for recursive process. Go to Step 2;

Step 8: Perform $3 \times 3$ mean filter for background clutter pixels in $M_{s i}$. And then obtain the resulting image by normalizing the $M_{s i}$ from 0 to 1 .

where $m$ is the weighting factor of discrimination for candidate target pixels and background clutter pixels and $m=0.7$ is used in experiment. In the above procedure, the summation image of the multi-SEs NWTH images in step 3 is used due to the following reason. The occurrence ratio of background clutter in the multi-SEs NWTH images may be different in same pixel coordinates due to those SE sizes. On the other hand, pixel values of small target region in the multi-SEs NWTH images can be strongly appeared regardless of those SE sizes, if a base SE is converged by recursive process. So the summation image of the multi-SE NWTH images has higher targetto-clutter ratio than a NWTH image by a particular SE. The mean filter in step 8 is used for clutter suppression in background region, and it makes real target pixels in background clutter pixels prominent.

\section{Experimental results}

Fig. 2 shows the simulation results from 3 IR images using the proposed recursive multi-SEs NWTH method. The first image (A) has a image of $360 \times 240$ size with cloud background and the second image (B) has a image of $320 \times 240$ size with high contrast, $10 \%$ Gaussian noises and the third image $(\mathrm{C})$ has a image of $320 \times 240$ size with low contrast. The initial size of the multi-SEs was set to $a=5, b=9, c=13$ and $s i=4$. Column (a) shows original IR images containing small targets in various backgrounds. Column (b), (c), and (d) show normalized images of respective result images by the WTH transformation, the NWTH transformation, and the proposed method. Experimental results show that the proposed method has superior target enhancement and background clutter suppression ability comparing to the WTH transformation and the NWTH transformation.

For the objective comparison of respective methods, two metrics, the signal-to-clutter ratio gain (SCRG) and the background suppression factor (BSF) [7], are employed and defined as the following:

$$
S C R=\frac{(S / C)_{\text {out }}}{(S / C)_{\text {in }}}, B S F=\frac{C_{\text {in }}}{C_{\text {out }}} .
$$

where $S$ is the target signal amplitude and $C$ is the clutter standard deviation in a normalized image. The experimental data using $\mathrm{A}, \mathrm{B}$, and $\mathrm{C}$ images are showed in Fig. 3. From both subjective and objective comparison results, 


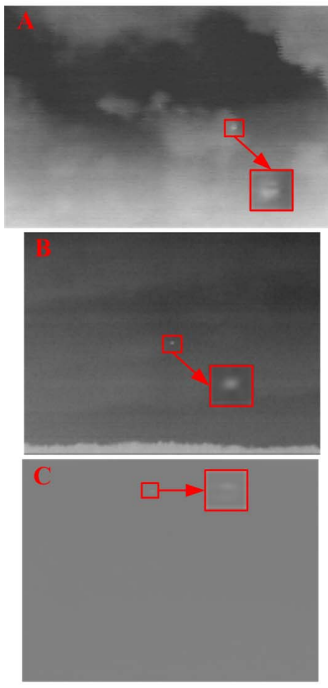

(a)

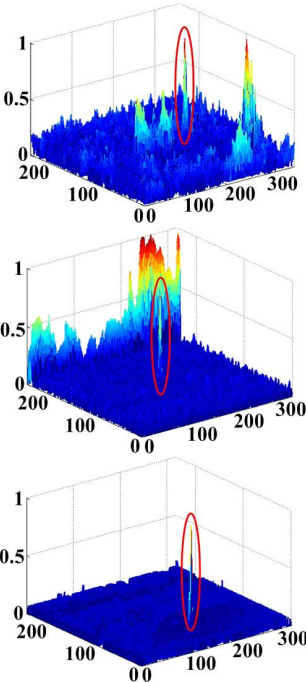

(b)
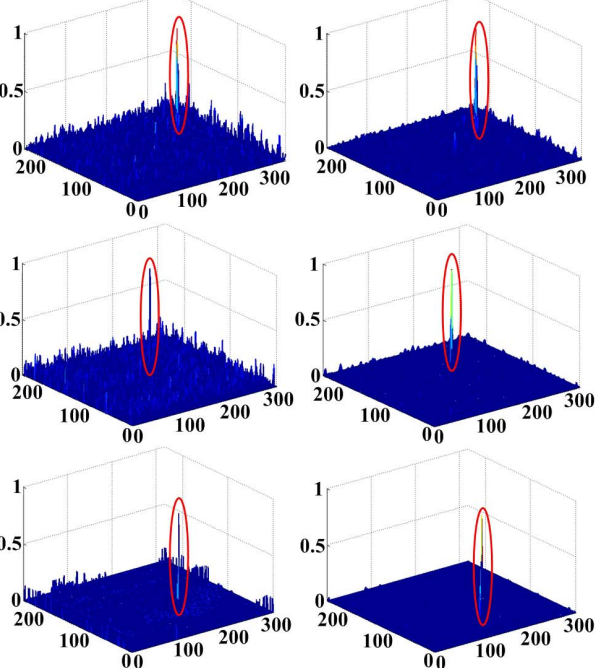

(c)

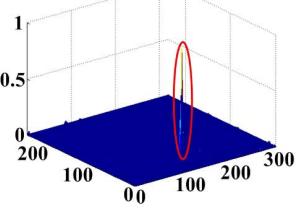

(d)

Fig. 2. Simulation results about 3 IR images. Column (a) Original IR images, Column (b) WTH transformation, Column (c) NWTH transformation, Column (d) Proposed recursive multi-SEs NWTH method.

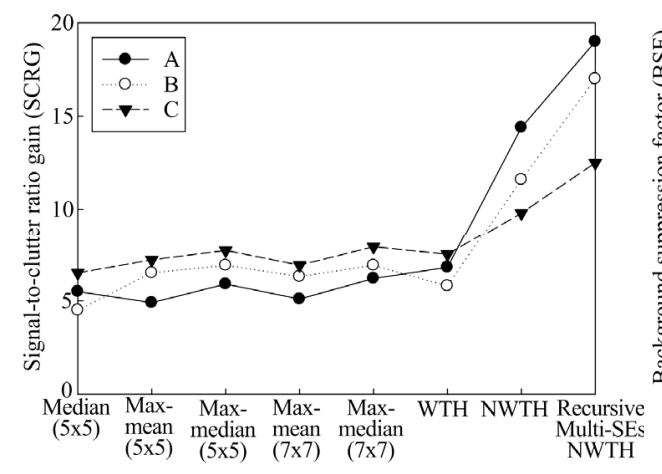

(a)

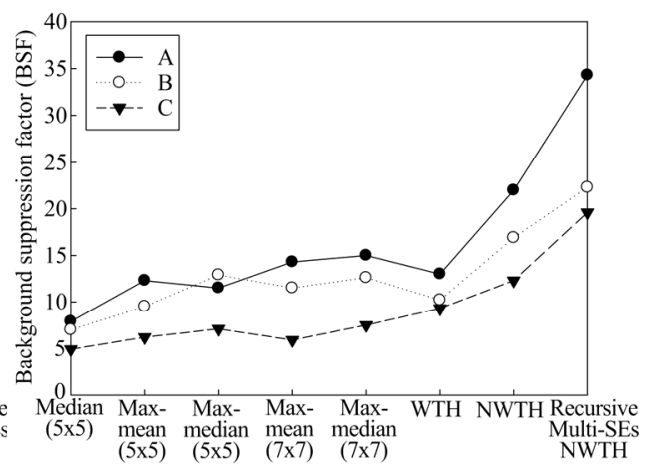

(b)

Fig. 3. Objective comparison with (a) SCRG and (b) BSF.

we can clearly see that the proposed recursive multi-SEs NWTH method has better performance for IR small target enhancement compared to the WTH transformation and the NWTH transformation.

\section{Conclusions}

In this paper, we propose a recursive multi-SEs NWTH method for small target enhancement. In IR target detection based on the morphological operator, the critical technology is the design of the morphological operator and the SE size. The proposed recursive multi-SEs NWTH method automatically selects the SE size by calculating the CTCRG of the NWTH images by the multi-SEs. Through the recursive process, the multi-SEs and SE interval is updated. The mentioned recursive process extracts the final multi-SEs

(c) IEICE 2011

DOI: 10.1587/elex.8.1576 Received August 06, 2011 Accepted September 08, 2011 Published October 10, 2011 
with the highest target enhancement ability. In the summation image of the multi-SEs NWTH images by final multi-SEs, small targets can easily be detected.

\section{Acknowledgments}

This work was supported by a basic research project of ADD (Agency for defense development) in Korea. 